\title{
Reduction of the integrated odd multipoles in periodic magnets
}

\author{
Simona Bettoni ${ }^{1,2}$ \\ ${ }^{1}$ CERN, Geneva, Switzerland \\ ${ }^{2}$ Universitá degli Studi di Milano, Milan, Italy \\ (Received 9 October 2006; published 30 April 2007)
}

\begin{abstract}
Integrated multipoles may perturb the beam dynamics in accelerators. In particular in periodic magnets, as undulators and wigglers, only the odd orders in the U.S. notation have to be considered, because the other ones compensate themselves in each period of the magnet. In the past, several methods have been considered to reduce them, but, if their effects on the beam are very strong, because of the small particles energy and mass, and in long period magnets, a different solution has to be explored. The method proposed in this article consists in compensating the contribution to the integral of the region between the poles with the one of the pole regions in each semiperiod of the magnet. It will be shown that this condition can be achieved by displacing the magnetic axis in each semiperiod of the magnet. To demonstrate the validity of this approach, it has been applied and optimized on a real wiggler.
\end{abstract}

DOI: 10.1103/PhysRevSTAB.10.042401

PACS numbers: $41.85 . \mathrm{Lc}$

\section{INTRODUCTION}

Integrated multipoles with respect to the beam trajectory may be a source of perturbation for the beam dynamics in accelerators [1]. In particular for periodic magnets, as undulators and wigglers, only the odd orders in the U.S. notation (quadrupole, octupole, dodecapole, ...) have to be considered, because the integrals of the even multipoles (dipole, sextupole, decapole, ...) are equal in magnitude and opposite in sign from a pole to the following one, so that they cancel themselves in each period.

Several approaches may be considered to reduce the integrated odd multipoles. A possibility consists in adding some jaws at the extremities of the magnet to compensate a specific order integrated multipole. This solution, already successfully applied in the past [2], may be decisive if only a specific order needs a correction and a small one, so that this approach can be done without significantly modifying the other orders. The improvement of the transverse field uniformity by means of pole shims allows to reduce all the odd integrated multipoles, but only in the pole regions. This solution may be therefore ineffective in magnets with short length poles, because of the contributions to the integrals from the regions between the poles, which are not reduced by this intervention.

The approach presented in this article is to compensate the contributions to the integrals of the multipoles from the pole regions with the ones of the regions between the poles by opportunely displacing the magnetic axis. It will be shown that in this way all the integrated odd multipoles are reduced with respect to a configuration with a fixed magnetic axis and that a specific odd order can be made ideally null.

In Sec. II the method is described and justified, and in Sec. III its application and optimization on a real magnet are presented.

\section{THE GENERAL METHOD}

All the analyses presented in this article have been done calculating the multipoles around the beam trajectory, but to prove the validity of the approach it is convenient to express these coefficients as a function of the ones calculated around the magnetic axis.

Let $z$ be the longitudinal axis of the magnet, $y$ the vertical dimension, and $x$ and $\tilde{x}$ the horizontal transverse coordinates with respect to $z$ and the beam trajectory, respectively. At each $\bar{z}$ the vertical component of the magnetic field, $B_{y}$, expanded around the beam trajectory $\left(x_{T R}, y_{T R}, \bar{z}\right)$, considering the motion in the midplane, is given by

$$
B_{y}(\tilde{x}, 0, \bar{z})=\left.\sum_{n=0}^{\infty} \frac{1}{n !} \cdot \frac{\partial^{n} B_{y}(\tilde{x}, 0, \bar{z})}{\partial \tilde{x}^{n}}\right|_{\tilde{x}=0} \cdot \tilde{x}^{n}
$$

where the derivatives are calculated on the beam trajectory. Introducing the coefficients $b_{n}{ }^{T}$, which correspond to the multipoles with respect to the beam trajectory $\left(b_{0}{ }^{T}\right.$ to the dipole, $b_{1}{ }^{T}$ to the quadrupole, ...), Eq. (1) becomes

$$
B_{y}(\tilde{x}, 0, \bar{z},) \equiv \sum_{n=0}^{\infty} b_{n}{ }^{T} \cdot \tilde{x}^{n} \text {. }
$$

The generic multipole with respect to the beam trajectory, $b_{n}^{T}$, as a function of the ones calculated around the magnetic axis, $b_{k}{ }^{A}$, is given by

$$
b_{n}{ }^{T}=\sum_{k=n}^{\infty} b_{k}{ }^{A} \cdot \frac{k !}{n ! \cdot(k-n) !} \cdot x_{\mathrm{TR}}{ }^{k-n} .
$$

neglecting the angle between $x$ and $\tilde{x}$. In particular an even term, $b_{2 \cdot j}{ }^{T}$, can be written as 


$$
\begin{aligned}
b_{2 \cdot j}{ }^{T}= & c_{2 \cdot j} \cdot b_{2 \cdot j}{ }^{A}+c_{2 \cdot j+1} \cdot b_{2 \cdot j+1}{ }^{A} \cdot x_{T R} \\
& +c_{2 \cdot j+2} \cdot b_{2 \cdot j+2}{ }^{A} \cdot x_{T R}^{2}+c_{2 \cdot j+3} \cdot b_{2 \cdot j+3}{ }^{A} \cdot x_{T R}^{3} \\
& +\cdots
\end{aligned}
$$

introducing the positive coefficients $c_{2 \cdot j}, c_{2 \cdot j+1}, \ldots$ Equation (4) proves that the integrals of the even orders with respect to the beam trajectory cancel themselves in each period of the magnet. The contributions of the even orders with respect to the magnetic axis, in fact, are equal in modulus and opposite in sign from a pole to the next one for the alternating sign of the current, so that they cancel themselves in each period, and the other terms are null at each $\bar{z}$ because of the left-right symmetry of the magnet. The generic odd term calculated with respect to the beam trajectory, $b_{2 \cdot j+1}{ }^{T}$, from Eq. (3) is instead given by

$$
\begin{aligned}
b_{2 \cdot j+1}{ }^{T}= & c_{2 \cdot j+1} \cdot b_{2 \cdot j+1}{ }^{A}+c_{2 \cdot j+2} \cdot b_{2 \cdot j+2}{ }^{A} \cdot x_{T R} \\
& +c_{2 \cdot j+3} \cdot b_{2 \cdot j+3}{ }^{A} \cdot x_{T R}^{2}+c_{2 \cdot j+4} \cdot b_{2 \cdot j+4}{ }^{A} \cdot x_{T R}^{3} \\
& +\cdots .
\end{aligned}
$$

In this case the odd order terms are still null, but the other ones are not, because both the even orders with respect to the magnetic axis and the odd powers of $x_{T R}$ change sign from a pole to the following one, so that their product sums itself in each period of the magnet. In particular $b_{3}{ }^{T}$, which will be considered in the next section for the application of the method, is given by the sum of the terms which contain the decapole and the higher even orders calculated with respect to the magnetic axis:

$$
b_{3}{ }^{T}=c_{4} \cdot b_{4}{ }^{A} \cdot x_{T R}+c_{6} \cdot b_{6}{ }^{A} \cdot x_{T R}^{3}+\cdots .
$$

The final scope of the method is to minimize the effect of the multipoles on the beam dynamics, described by their integral along the entire magnet, $I_{n}$ :

$$
I_{n} \equiv \int_{\text {magnet }} b_{n}^{T} \cdot d z \quad n=0,1,2, \ldots
$$

In particular the integrals $I_{2 \cdot j+1}$ of the generic odd multipoles $b_{2 \cdot j+1}{ }^{T}$ are given by

$$
\begin{aligned}
I_{2 \cdot j+1}= & \int\left(c_{2 \cdot j+2} \cdot b_{2 \cdot j+2}{ }^{A} \cdot x_{T R}\right. \\
& \left.+c_{2 \cdot j+4} \cdot b_{2 \cdot j+4}{ }^{A} \cdot x_{T R}^{3}+\cdots\right) \cdot d z .
\end{aligned}
$$

The method consists in displacing the magnetic axis in the poles to change the sign of $x_{T R}$ in Eq. (8), so that the integral of a generic odd multipole can be made null in each semiperiod of the magnet.
In Fig. 1 the beam trajectory in the $z-x$ plane in a semiperiod of a magnet is schematically represented. Without any shaping of the pole the geometric and the magnetic axes, indicated as G.A. and M.A. respectively, coincide, so that the sign of $x_{T R}$ does not change along the entire semiperiod.

If the magnetic axis is displaced from the geometric one, the beam trajectory is on the left of the magnetic axis for a part of the motion (Fig. 2), and on the right for the rest of the semiperiod (Fig. 3), so that $x_{T R}$ changes sign.

Opportunely choosing the position of the magnetic axis, therefore, an integrated odd multipole can be made ideally null. Furthermore because Eq. (8) is valid for a generic odd integrated multipole, all the odd orders are simultaneously reduced by this method.

This approach has been applied to the wiggler of the main ring of DAФNE (Frascati, INFN Laboratory) to make null the integrated octupole with respect to the beam trajectory. In Sec. III the procedure will be described in detail.

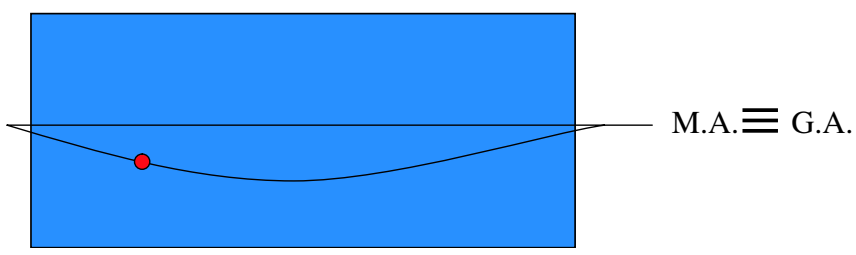

FIG. 1. (Color) Beam trajectory in a semiperiod of the magnet without any shaping of the pole. In this case the geometric (G.A.) and the magnetic (M.A.) axes coincide.

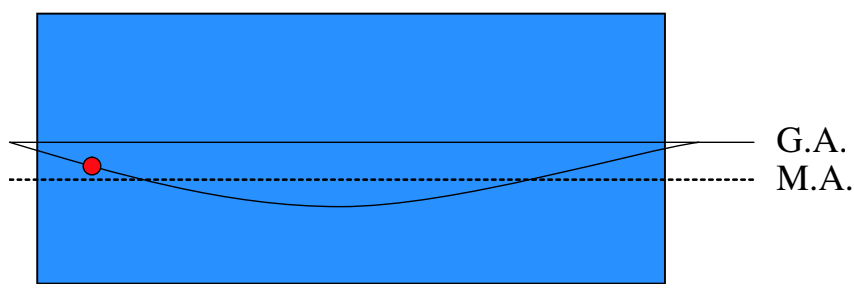

FIG. 2. (Color) Beam trajectory in a semiperiod of the magnet. The geometric (G.A.) and the magnetic (M.A.) axes of the wiggler are separated. In this case the particle is at the left of the magnetic axis.

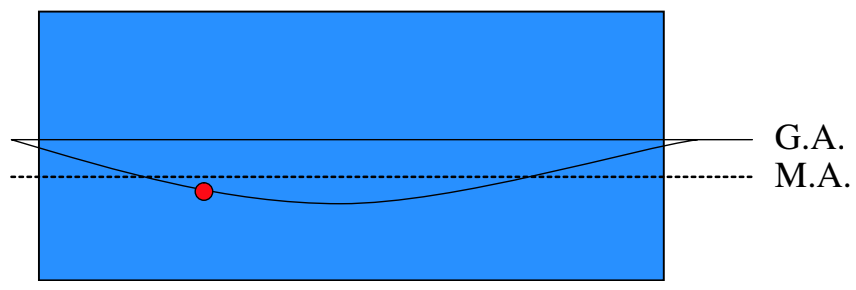

FIG. 3. (Color) Beam trajectory in a semiperiod of the magnet. The geometric (G.A.) and the magnetic (M.A.) axes of the wiggler are separated. In this case the particle is at the right of the magnetic axis. 


\section{THE APPLICATION TO THE WIGGLER OF THE MAIN RING OF DAФNE}

In the main ring of DAФNE (Frascati, INFN Laboratory) eight normal conducting wigglers have been installed to reduce the damping time. Since their first operation these magnets were sources of nonlinearity for the beam dynamics [3], because of the field roll-off combined with the large excursion of the particles from the axis of the magnet (amplitude of about $1.3 \mathrm{~cm}$ ), determined by the characteristics of the wigglers and the mass and the energy of the beams, summarized in Table I.

In the past several interventions oriented to improve the horizontal transverse field uniformity have been implemented. At present a non-negligible parabolic tune shift with respect to the horizontal displacement of the beam, due to the integrated octupole with respect to the beam trajectory, still persists [4]. The approach presented in the previous section has been applied to these wigglers to make null this integrated multipole.

As a preliminary step, the analysis of the wigglers in the present configuration has been done (Sec. III A) to be compared with the results of the optimization (Sec. III B).

\section{A. The analysis of the present situation}

In Fig. 4 the section in the $z-x$ plane of one of the wigglers of DA $\Phi N E$ is shown.

TABLE I. Wiggler specifications, particles, and energy beams circulating in the main ring of DAФNE.

\begin{tabular}{lcc}
\hline \hline & Full pole & Half pole \\
\hline Number of poles & 5 & 2 \\
Current (A) & 693 & 564 \\
Peak field (T) & $\sim 1.8$ & $\sim 1.5$ \\
Period (cm) & 64 & \\
Gap (cm) & 3.7 \\
Pole width (cm) & \multicolumn{2}{c}{14} \\
Particles beams & Electrons and positrons \\
Energy of the beams (MeV) & \multicolumn{2}{c}{510} \\
\hline \hline
\end{tabular}

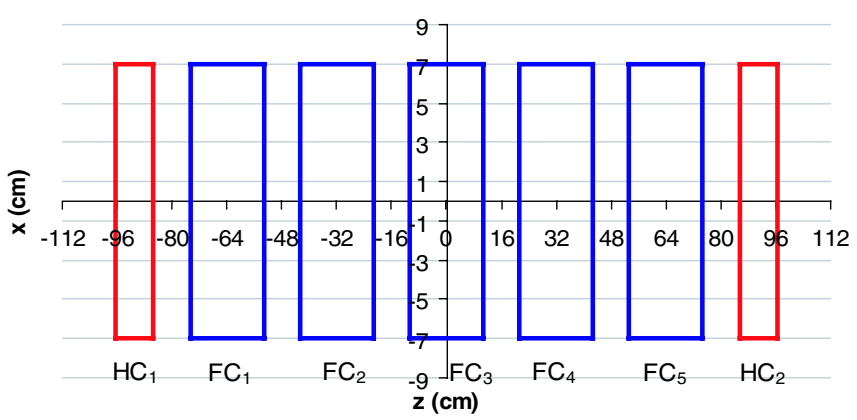

FIG. 4. (Color) Scheme of one of the wigglers of DAФNE in the $z-x$ plane in the present configuration.
The analysis of the present situation has been performed starting from an experimental map (measured by steps of $1 \mathrm{~cm}$ from $-1.35 \mathrm{~m}$ to $+1.35 \mathrm{~m}$ with respect to the center of the wiggler in $z$ and for the entire pole width in $x$ with the same step) of the vertical component of the magnetic field in the midplane, $B_{y}(x, 0, z)$, given by Miro Preger.

For each $\bar{z}$ the values of $B_{y}(\tilde{x}, 0, \bar{z})$ around the beam trajectory have been fitted as a function of $\tilde{x}$ in the range of $\pm 3 \mathrm{~cm}$ by a fourth order polynomial determined from considerations about the goodness of the fit:

$$
\begin{aligned}
B_{y}(\tilde{x}, 0, \bar{z})= & b_{0}{ }^{T}+b_{1}{ }^{T} \cdot \tilde{x}+b_{2}{ }^{T} \cdot \tilde{x}^{2}+b_{3}{ }^{T} \cdot \tilde{x}^{3} \\
& +b_{4}{ }^{T} \cdot \tilde{x}^{4} .
\end{aligned}
$$

The integrals defined in Eq. (7) calculated in the central semiperiod and in the entire magnet are shown in Table II, and, in particular, in Fig. 5 the third order term of the fit as a function of $z$ is plotted.

The anomalous behavior of the second and the fourth orders, which do not cancel along the entire magnet and the asymmetry of $b_{3}{ }^{T}$ as a function of $z$ may be imputed to a modification made only on one of the terminal poles to improve the dynamic aperture of the ring [4].

The values in Table II will be compared with the results of the optimization in the next section.

TABLE II. Present situation: integrated coefficients of the transversal expansion of the magnetic field in the central semiperiod and in the entire wiggler.

\begin{tabular}{lrrrrr}
\hline \hline & $I_{0}(\mathrm{~T} \mathrm{~m})$ & $I_{1}(\mathrm{~T})$ & $I_{2}(\mathrm{~T} / \mathrm{m})$ & $I_{3}\left(\mathrm{~T} / \mathrm{m}^{2}\right)$ & $I_{4}\left(\mathrm{~T} / \mathrm{m}^{3}\right)$ \\
\hline $\pm 16 \mathrm{~cm}$ & -0.40 & -0.09 & 0.80 & -26.5 & 1448.7 \\
Whole & 0.00 & -0.09 & -3.35 & -139.7 & 1772.2 \\
\hline \hline
\end{tabular}

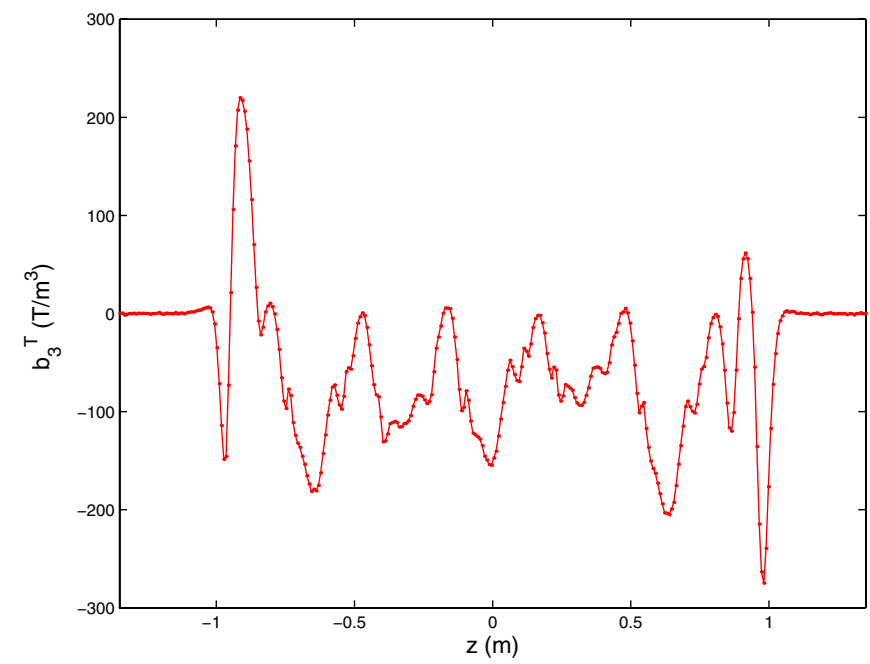

FIG. 5. (Color) Third order term of the transversal expansion of the magnetic field calculated with respect to the beam trajectory as a function of $z$ in the configuration at present. 


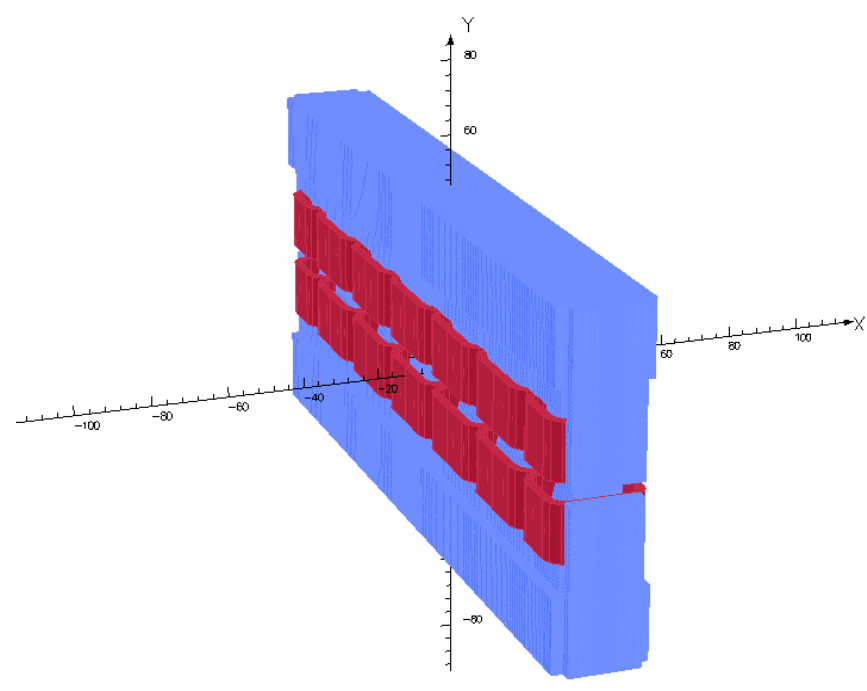

FIG. 6. (Color) 3D model of the complete wiggler of DAФNE without any shaping of the poles. For the symmetry of the wiggler only $1 / 8$ of the entire magnet has been simulated. Dimensions are in $\mathrm{cm}$.

\section{B. The modification of the wiggler}

The optimization has been done in three steps. The first one was the determination of the best position of the magnetic axis to minimize the integrated octupole. As the second step the dependence of the solution on the beam-wiggler misalignment has been reduced by adding pole shims. Because these shims modify also the optimal position of the magnetic axis, it has been re-optimized in the new and definitive configuration.

The wiggler in the present configuration cannot be easily simulated, because of the numerous modifications made in the past to improve the transverse field uniformity, therefore a preliminary $3 \mathrm{D}$ model $^{1}$ of the magnet without any shaping of the poles, shown in Fig. 6, has been used to validate the model by comparing the results of the simulation with the experimental measurements made before any intervention on the magnet (flat poles) [4].

The magnetic axis can be displaced by several ways. In the case of superconducting magnets, this condition can be obtained by moving the coils in the transverse dimension [6], whereas in the case of normal conducting magnets it is preferable to modify the poles.

To avoid that for this intervention the integrated even multipoles with respect to the beam trajectory are increased, the even terms with respect to the magnetic axis have to be maintained equal in magnitude and opposite in sign from a pole to the next one, and the odd orders with respect to the magnetic axis must remain null according to Eq. (4).

\footnotetext{
${ }^{1}$ All the 3D magnetic models have been done with OPERA Vector Fields analysis [5].
}

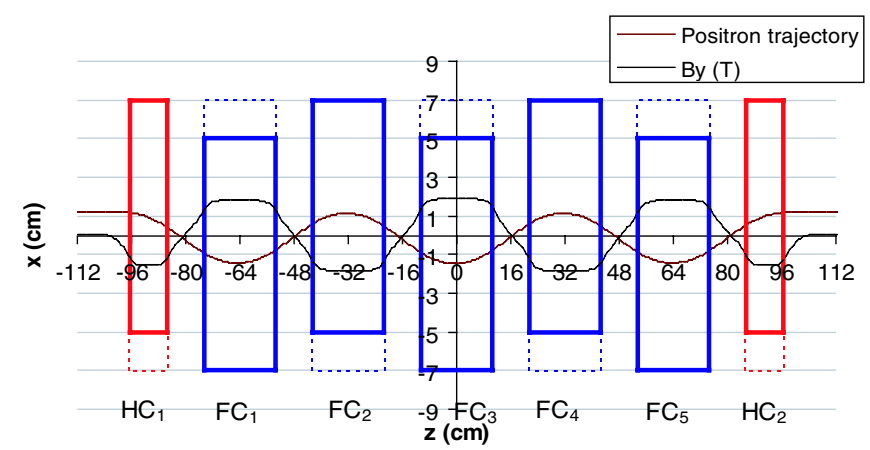

FIG. 7. (Color) Section in the $z-x$ plane of the modified wiggler (solid line). The configuration of the poles before any modification is also shown (dashed lines).

The first condition is achieved by modifying the poles in such a way that the magnetic axis only reverses sign from a pole to the next one, as sketched in Fig. 7.

To satisfy the second request the shape of the poles in the $x-y$ plane has to preserve the left-right symmetry with respect to the new magnetic axis. In Fig. 8 the section of the equivalent 2D model of a pole in the $x-y$ plane before any modification is shown.

In Fig. 9 the shape of the poles in the same plane after the modification is shown. To have a perfect left-right symmetry, the cut should reach the top of the yoke, but the team of Frascati asked to modify only the pole. In any

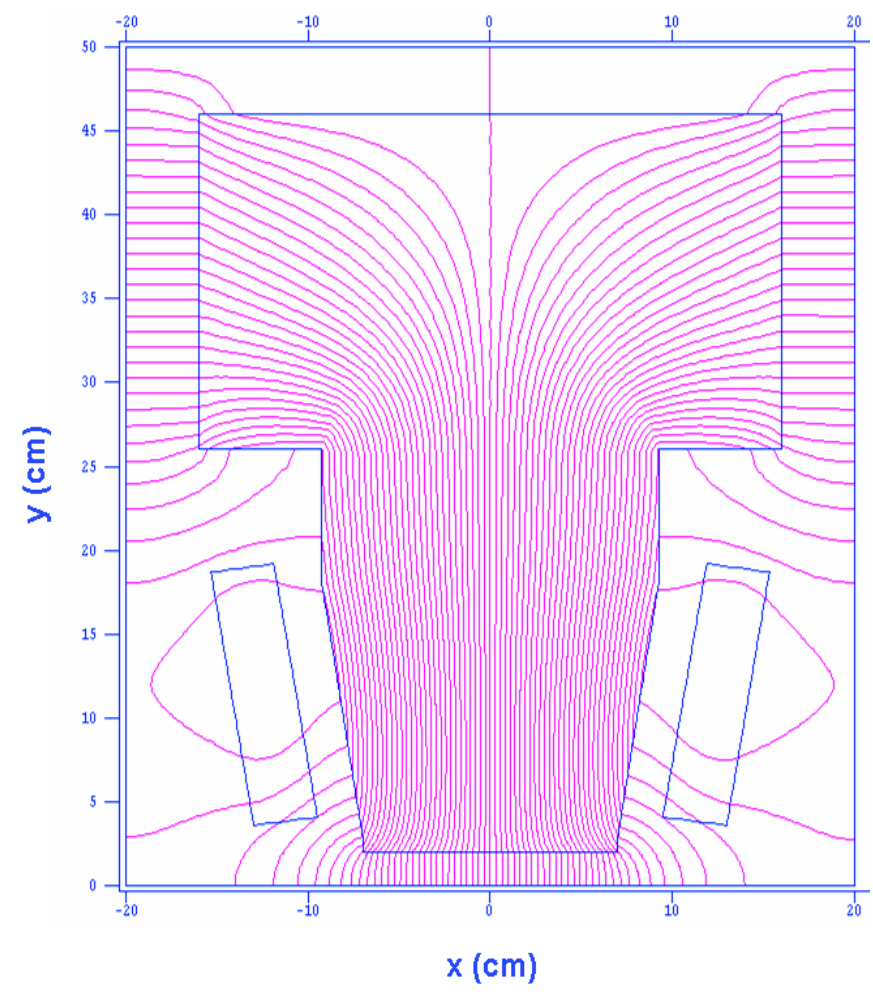

FIG. 8. (Color) Section in the $x-y$ plane of the equivalent model of the pole of the wiggler before any modification. 

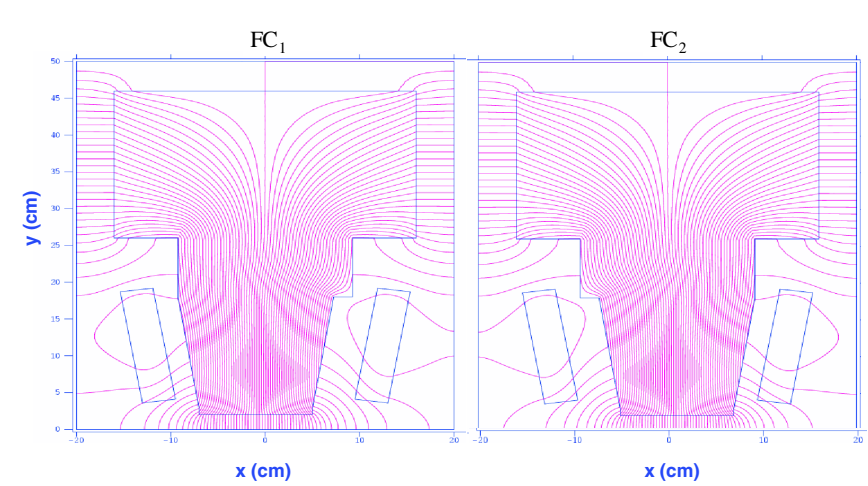

FIG. 9. (Color) Sections in the $x-y$ plane of the poles after the modification in case the beam trajectory is at negative $\left(\mathrm{FC}_{1}, \mathrm{FC}_{3}\right.$, and $\left.\mathrm{FC}_{5}\right)$ or positive $\left(\mathrm{FC}_{2}, \mathrm{FC}_{4}, \mathrm{HC}_{1}\right.$, and $\left.\mathrm{HC}_{2}\right) x$.

case this approximation seems to be reasonable if considering that the cut reaches about $20 \mathrm{~cm}$ above the midplane.

The average beam trajectory in the pole regions, indicated in Table III, has been chosen as the starting new position of the magnetic axis. The poles have been therefore cut symmetrically to $x=-1 \mathrm{~cm}$ in $\mathrm{FC}_{1}\left(\mathrm{FC}_{3}\right.$ and $\mathrm{FC}_{5}$ ), where the beam trajectory is at negative $x$, and to $x=+1 \mathrm{~cm}$ in $\mathrm{FC}_{2}\left(\mathrm{FC}_{4}, \mathrm{HC}_{1}\right.$, and $\left.\mathrm{HC}_{2}\right)$, where the beam trajectory is at positive $x$. The sketch of the poles in the $z-x$ plane after the modification in the two cases is shown in Fig. 10.

The running time of a simulation of the $3 \mathrm{D}$ magnetic model of the complete wiggler with the cut poles would be very long, because to obtain a meaningful fit of the magnetic field for the analysis the number of nodes has to be large, and moreover because $1 / 4$ and not only $1 / 8$ of the wiggler has to be designed for the asymmetry introduced by the cut with respect to the $z-y$ plane. The optimization has hence been done on the central semiperiod (from $z=-16 \mathrm{~cm}$ to $z=+16 \mathrm{~cm}$ ) using an approximated short model of the central part of the wiggler (central full pole and the two closest ones with the opportune boundary conditions to simulate $\mathrm{FC}_{2}, \mathrm{FC}_{3}$, and $\mathrm{FC}_{4}$ ) shown in Fig. 11. Only after the results on the central semiperiod of the wiggler have been considered satisfactory, the model of the complete wiggler and the calculations have been done on the other semiperiods.

In Fig. 12 the section of the short model in the $z-x$ plane is shown.

TABLE III. Average beam trajectory in the poles of the wiggler at positive $z$.

\begin{tabular}{lc}
\hline \hline Pole & Average beam trajectory $(\mathrm{cm})$ \\
\hline $\mathrm{FC}_{3}$ & -0.97 \\
$\mathrm{FC}_{4}$ & 1.04 \\
$\mathrm{FC}_{5}$ & -1.01 \\
$\mathrm{HC}_{2}$ & 0.95 \\
\hline \hline
\end{tabular}
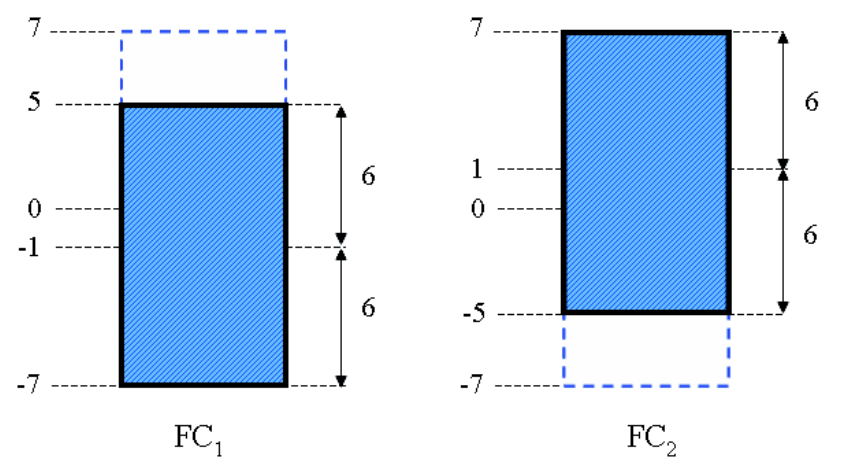

FIG. 10. (Color) Sketch in the $z-x$ plane of the poles cut symmetrically with respect to the average beam trajectory for $\mathrm{FC}_{1}$ $\left(\mathrm{FC}_{3}\right.$ and $\left.\mathrm{FC}_{5}\right)$ and for $\mathrm{FC}_{2}\left(\mathrm{FC}_{4}, \mathrm{HC}_{1}\right.$, and $\left.\mathrm{HC}_{2}\right)$. The shape of the poles without any modification is also indicated (dashed line). Dimensions are in $\mathrm{cm}$.

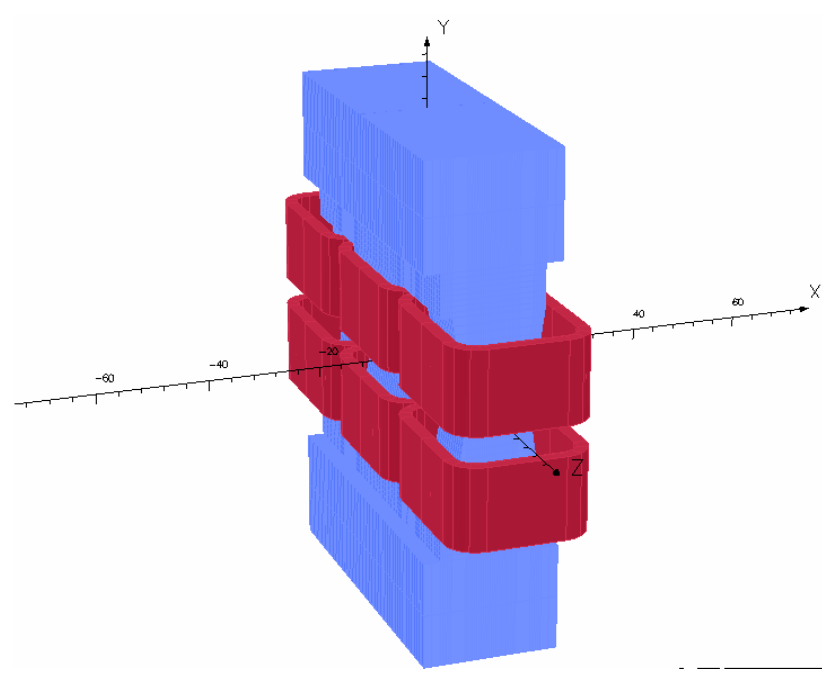

FIG. 11. (Color) Short model of the wiggler. Dimensions are in $\mathrm{cm}$.

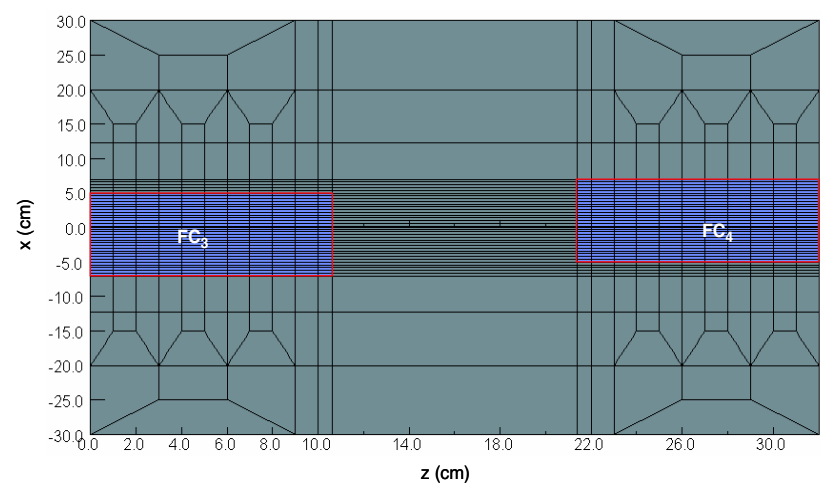

FIG. 12. (Color) Section in the $z-x$ plane of the short model of the wiggler for the preliminary optimization. The steps in the region of the poles are of $1 \mathrm{~cm}$ in $z$ and $0.4 \mathrm{~cm}$ in $x$. The structure of trapezes from $x= \pm 12.25 \mathrm{~cm}$ to $x= \pm 30 \mathrm{~cm}$ is to have a high number of nodes in the region of the fit without unnecessarily increasing the subdivisions at the boundary of the model. 
TABLE IV. Poles cut symmetrically to the average beam trajectory in the pole regions (short model): integrated coefficients of the polynomial expansion of the magnetic field.

\begin{tabular}{lrrrrr}
\hline \hline & $I_{0}(\mathrm{~T} \mathrm{~m})$ & $I_{1}(\mathrm{~T})$ & $I_{2}(\mathrm{~T} / \mathrm{m})$ & $I_{3}\left(\mathrm{~T} / \mathrm{m}^{2}\right)$ & $I_{4}\left(\mathrm{~T} / \mathrm{m}^{3}\right)$ \\
\hline Present & -0.40 & -0.09 & 0.80 & -26.5 & 1448.7 \\
Model & 0.43 & -0.05 & -7.62 & -1.44 & -3141.6 \\
\hline \hline
\end{tabular}

As for the analysis of the present situation, the vertical component of the magnetic field has been fitted for each $\bar{z}$ by a fourth order polynomial in the range of $\pm 3 \mathrm{~cm}$ around the beam trajectory and the integrals defined in Eq. (7) have been calculated in the central semiperiod of the magnet.

As it can be seen from the comparison of the analysis of the field map of the model with the present situation, shown in Table IV, the integral $I_{3}$ is strongly reduced by this intervention. This is obtained because the contributions to the integrated octupole compensate themselves along the semiperiod for the alternating sign of $b_{3}{ }^{T}$ as a function of $z$, as evident in Fig. 13.

In particular in Fig. 14 it can be seen that the sign of $b_{3}{ }^{T}$ reverses at the points where the beam trajectory intersects the magnetic axis.

Because of the satisfactory factor of reduction of $I_{3}$ in the central semiperiod, this configuration has been used to make the model of the complete magnet.

The integrated coefficients of the field expansion calculated in the central semiperiod from the model of the complete wiggler compared with the present situation are indicated in Table V. The comparison between Table IV and Table $\mathrm{V}$ shows that the integral of $b_{3}{ }^{T}$ is higher than $I_{3}$ calculated from the short model, but the factor of reduction

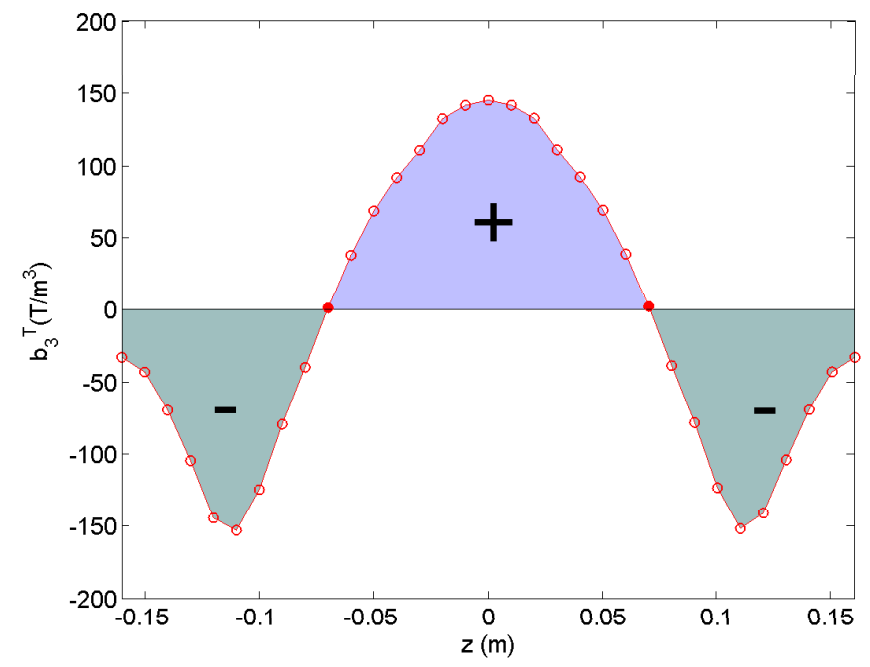

FIG. 13. (Color) Third order coefficient of the transverse expansion of the magnetic field along the beam trajectory in the central semiperiod as a function of $z$. The part of the plane which gives positive and negative contributions to $I_{3}$ and the points corresponding to the inversion of sign of $b_{3}{ }^{T}$ are put in evidence.

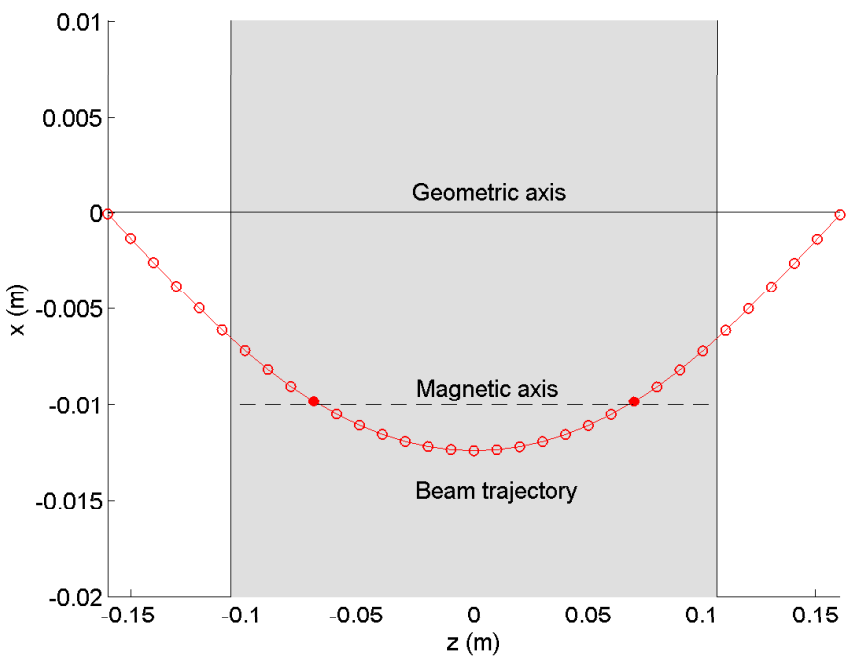

FIG. 14. (Color) Beam trajectory in the central semiperiod as a function of $z$. The points corresponding to the intersection of the beam trajectory and the new magnetic axis are put in evidence.

is about 10 with respect to the present situation, so that this configuration has been used for the second step of the optimization: the dependence of $I_{3}$ on a beam-wiggler misalignment.

To study this aspect the $I_{n}$ in the central semiperiod have been calculated for $x$-shifts of the beam of $\pm 2 \mathrm{~mm}$ at the entrance of the wiggler with respect to the nominal trajectory.

As it can be seen in Table VI, the dependence of the solution on the beam-wiggler misalignment may have catastrophic effects on the results (a $x$-shift of only $2 \mathrm{~mm}$ destroys the advantage of the displacement of the magnetic axis).

To reduce this effect, pole shims have therefore been added to improve the transverse field uniformity in the pole regions. The drawing of a pole with the optimized shims in

TABLE V. Poles cut around the average beam trajectory in the pole regions (complete model): integrated coefficients of the polynomial expansion of the magnetic field.

\begin{tabular}{lrrrrr}
\hline \hline & $I_{0}(\mathrm{~T} \mathrm{~m})$ & $I_{1}(\mathrm{~T})$ & $I_{2}(\mathrm{~T} / \mathrm{m})$ & $I_{3}\left(\mathrm{~T} / \mathrm{m}^{2}\right)$ & $I_{4}\left(\mathrm{~T} / \mathrm{m}^{3}\right)$ \\
\hline Present & -0.40 & -0.09 & 0.80 & -26.5 & 1448.7 \\
Model & 0.42 & -0.05 & -7.29 & -2.62 & -3290.8 \\
\hline \hline
\end{tabular}

TABLE VI. Poles cut around the average beam trajectory: integrated coefficients of the field expansion as a function of the beam-wiggler misalignment in the central semiperiod.

\begin{tabular}{lccccc}
\hline \hline$x$-shift & $I_{0}(\mathrm{~T} \mathrm{~m})$ & $I_{1}(\mathrm{~T})$ & $I_{2}(\mathrm{~T} / \mathrm{m})$ & $I_{3}\left(\mathrm{~T} / \mathrm{m}^{2}\right)$ & $I_{4}\left(\mathrm{~T} / \mathrm{m}^{3}\right)$ \\
\hline$-2 \mathrm{~mm}$ & 0.42 & -0.08 & -7.37 & -30.15 & -3315.7 \\
Aligned & 0.42 & -0.05 & -7.29 & -2.62 & -3290.8 \\
$+2 \mathrm{~mm}$ & 0.43 & -0.02 & -7.34 & 24.79 & -3313.2 \\
\hline \hline
\end{tabular}




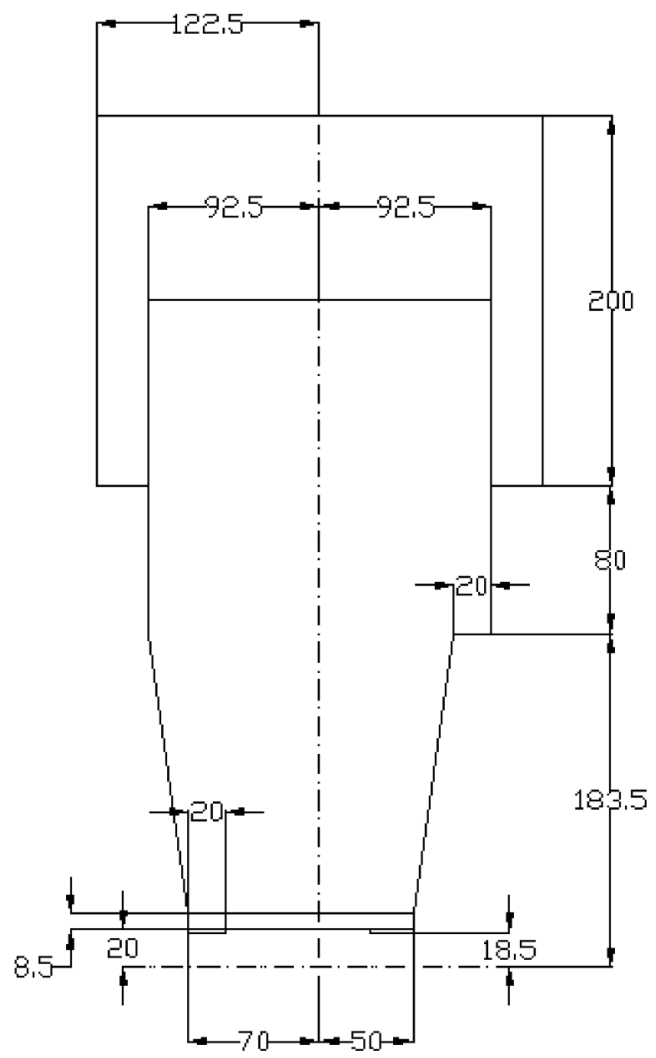

FIG. 15. Drawing in the $x-y$ plane of a pole of the wiggler with also the optimized shims. Dimensions are in $\mathrm{mm}$.

the $x-y$ plane is shown in Fig. 15, whereas in Fig. 16 a view of the model in the $z-x$ plane is shown.

In this configuration the integrated $b_{3}{ }^{T}$ calculated in the central semiperiod is much less dependent on the beamwiggler misalignment, as evident from the $I_{3}$ as a function of the $x$-shifts at the entrance of the wiggler, shown in Table VII.

In particular in Fig. 17 the absolute value of $I_{3}$ as a function of the horizontal beam-wiggler misalignment with and without the shims is plotted.

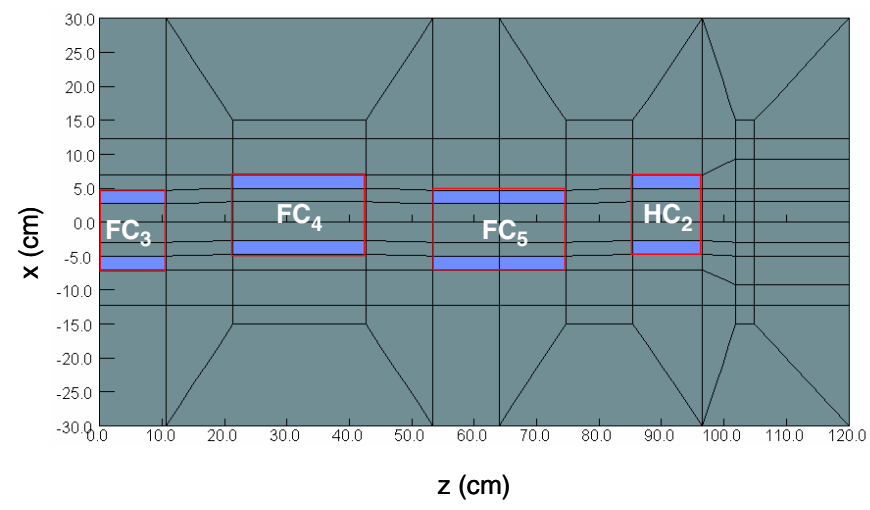

FIG. 16. (Color) Section in the $z-x$ plane of the model of the wiggler with also the optimized shims.
TABLE VII. Poles cut around the average beam trajectory in the pole regions with shims: integrated coefficients of the field expansion as a function of the beam-wiggler misalignment in the central semiperiod.

\begin{tabular}{cccccc}
\hline \hline$x$-shift & $I_{0}(\mathrm{~T} \mathrm{~m})$ & $I_{1}(\mathrm{~T})$ & $I_{2}(\mathrm{~T} / \mathrm{m})$ & $I_{3}\left(\mathrm{~T} / \mathrm{m}^{2}\right)$ & $I_{4}\left(\mathrm{~T} / \mathrm{m}^{3}\right)$ \\
\hline$-2 \mathrm{~mm}$ & 0.42 & -0.08 & -7.57 & -1.44 & -670.7 \\
Aligned & 0.42 & -0.05 & -7.50 & -6.70 & -596.9 \\
$+2 \mathrm{~mm}$ & 0.42 & -0.02 & -7.43 & -12.25 & -578.1 \\
\hline \hline
\end{tabular}

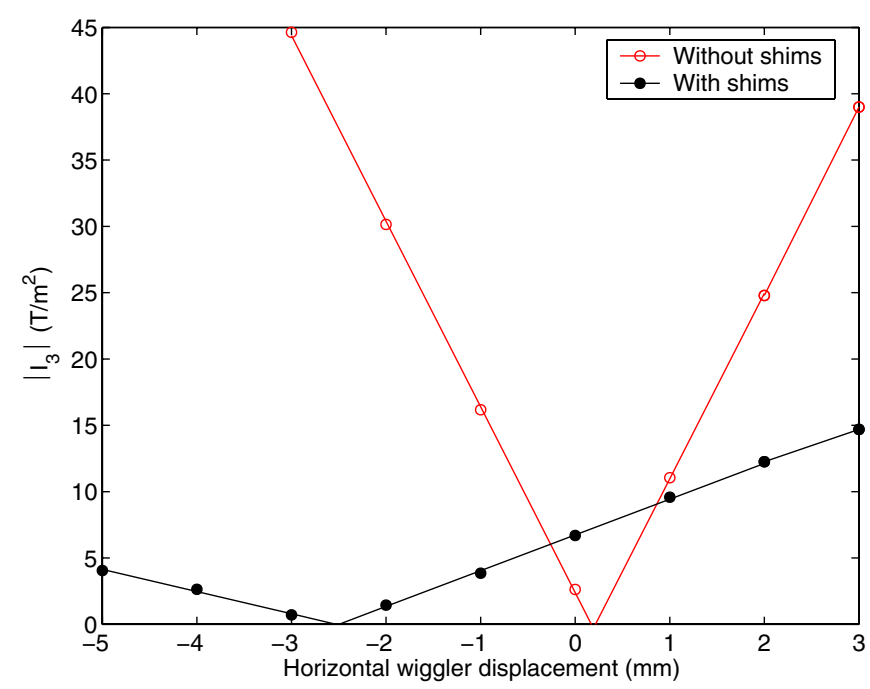

FIG. 17. (Color) Absolute value of $I_{3}$ calculated in the central semiperiod as a function of the beam-wiggler misalignment in the configuration with and without the shims.

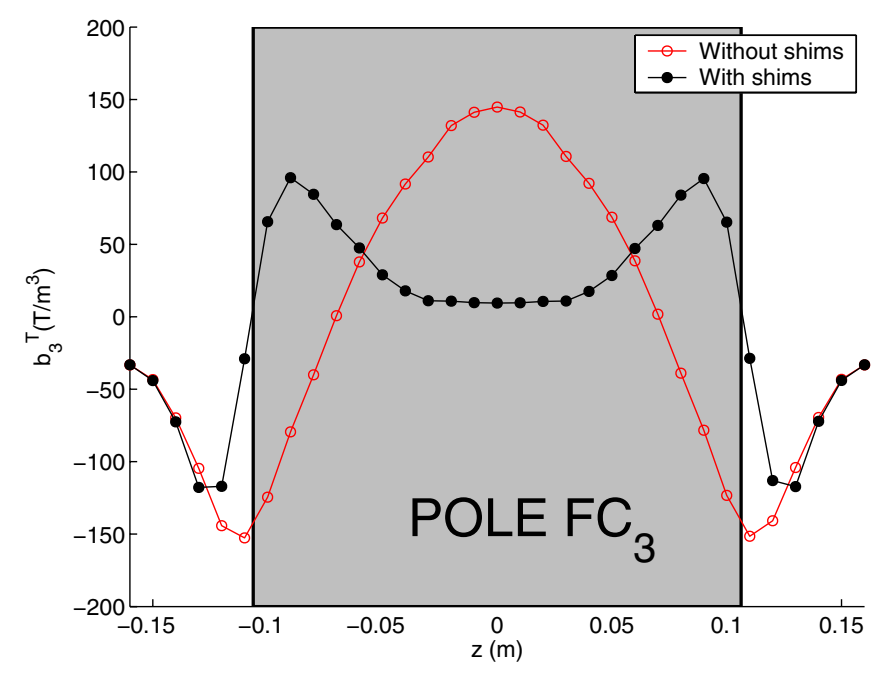

FIG. 18. (Color) Third order term of the field expansion as a function of $z$ without and with the shims.

The minimum of the absolute value of $I_{3}$ is displaced with respect to the condition of perfect beam-wiggler alignment $(x$-shift $=0 \mathrm{~mm})$, because the value of $b_{4}{ }^{A}$ is modified for the different saturation of the two shims as a 
function of $z$, caused by the not perfect left-right symmetry around the magnetic axis. Because of this effect, in fact, the values of $b_{3}{ }^{T}$ in the pole region and close to it are modified, as evident in Fig. 18.

The position of the magnetic axis has therefore been slightly modified to compensate this effect as a last step of the optimization.

The best position of the magnetic axis in the configuration with the shims is $-1.15 \mathrm{~cm}$ for $\mathrm{FC}_{1}, \mathrm{FC}_{3}$, and $\mathrm{FC}_{5}$, and $+1.15 \mathrm{~cm}$ for the other poles. In Table VIII the $I_{n}$ integrated in the central semiperiod for several $x$-shifts in this configuration are shown.

This configuration allows to maintain the slight dependence of the solution from the beam-wiggler misalignment, but also to displace the minimum of the absolute value of $I_{3}$ at $x$-shift $=0 \mathrm{~mm}$, as evident in Fig. 19.

The simulations indicate that the modification of the wiggler of DAФNE according to the configuration Cut 2 allows to reduce of 2 orders of magnitude $I_{3}$ in the central semiperiod with respect to the present situation, maintain-

TABLE VIII. Definitive configuration (central semiperiod): integrated coefficients of the polynomial expansion of the magnetic field.

\begin{tabular}{lccccc}
\hline \hline$x$-shift & $I_{0}(\mathrm{~T} \mathrm{~m})$ & $I_{1}(\mathrm{~T})$ & $I_{2}(\mathrm{~T} / \mathrm{m})$ & $I_{3}\left(\mathrm{~T} / \mathrm{m}^{2}\right)$ & $I_{4}\left(\mathrm{~T} / \mathrm{m}^{3}\right)$ \\
\hline$-3 \mathrm{~mm}$ & 0.42 & -0.12 & -7.87 & 6.30 & 708.9 \\
$-2 \mathrm{~mm}$ & 0.42 & -0.10 & -7.96 & 5.03 & 816.6 \\
$-1 \mathrm{~mm}$ & 0.42 & -0.09 & -8.0 & 2.86 & 867.7 \\
Aligned & 0.42 & -0.07 & -8.0 & -0.29 & 921.8 \\
$1 \mathrm{~mm}$ & 0.42 & -0.06 & -8.05 & -3.89 & 958.5 \\
$2 \mathrm{~mm}$ & 0.42 & -0.04 & -8.04 & -7.67 & 962.5 \\
$3 \mathrm{~mm}$ & 0.42 & -0.02 & -8.00 & -11.45 & 948.2 \\
\hline \hline
\end{tabular}

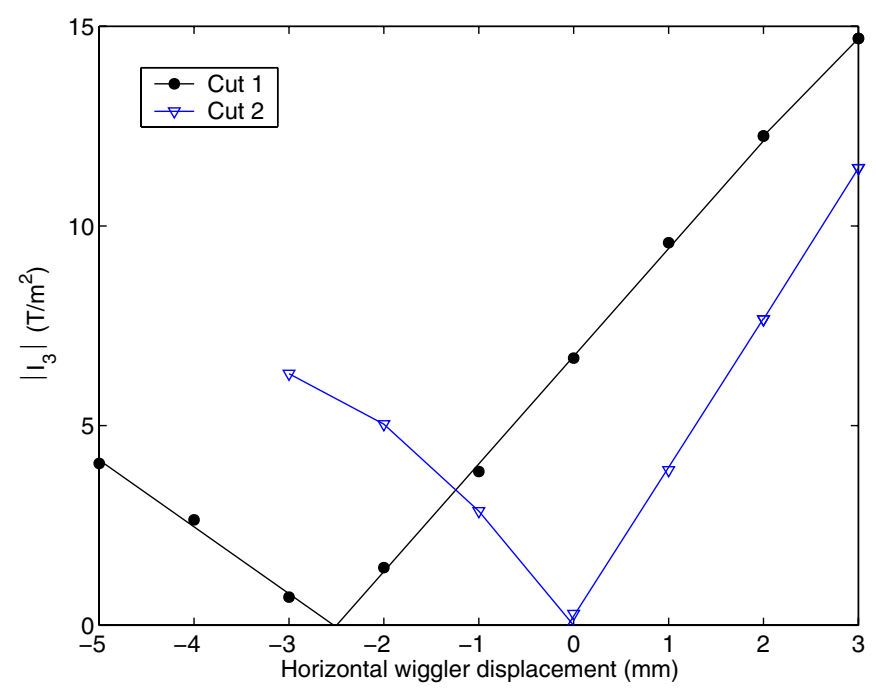

FIG. 19. (Color) Absolute value of $I_{3}$ in the central semiperiod as a function of the horizontal beam-wiggler misalignment in case the magnetic axis is displaced of $\pm 1 \mathrm{~cm}$ (Cut 1) and $\pm 1.15 \mathrm{~cm}$ (Cut 2) with respect to the geometric one.
TABLE IX. Definitive configuration (entire wiggler): integrated coefficients of the field expansion for the poles at positive $z$, except for $\mathrm{FC}_{3}$ where $b_{3}{ }^{T}$ is calculated from $z=-16 \mathrm{~cm}$ to $z=+16 \mathrm{~cm} . I_{3}$ calculated in the entire wiggler is not exactly the double of the sum of the contributions of $I_{3}$ at positive $z$, because of a small asymmetry of the beam trajectory in the two halves of the wiggler (the magnetic fields are equal for the symmetry of the model).

\begin{tabular}{lrrrrc}
\hline \hline & $I_{0}(\mathrm{~T} \mathrm{~m})$ & $I_{1}(\mathrm{~T})$ & $I_{2}(\mathrm{~T} / \mathrm{m})$ & $I_{3}\left(\mathrm{~T} / \mathrm{m}^{2}\right)$ & $I_{4}\left(\mathrm{~T} / \mathrm{m}^{3}\right)$ \\
\hline $\mathrm{FC}_{3}$ & 0.42 & -0.07 & -8.04 & -0.29 & 921.8 \\
$\mathrm{FC}_{4}$ & -0.42 & -0.07 & 8.07 & -0.47 & -904.1 \\
$\mathrm{FC}_{5}$ & 0.42 & -0.06 & -8.03 & -0.16 & 918.99 \\
$\mathrm{HC}_{2}$ & -0.21 & -0.03 & 2.99 & -1.53 & -657.1 \\
Whole & 0.00 & -0.36 & -1.67 & -4.13 & -327.1 \\
\hline \hline
\end{tabular}

ing an acceptable dependence on a wiggler-beam misalignment. The analysis has therefore been repeated on the other poles of the wiggler to determine their contributions to $I_{3}$.

In Table IX the $I_{n}$ integrated in the poles at positive $z$ are shown. The contributions of the full pole semiperiods to the integrals $I_{n}$ for all the orders are very similar to the one of the central semiperiod, whereas the terminal pole $\mathrm{HC}_{2}$ differs from them, because its length and saturation are not the same. In particular in Fig. 20 the third order term of the field expansion as a function of $z$ of the semiperiods at positive $z$ is plotted.

The simulations indicate that $I_{3}$ integrated in the complete wiggler is the $3 \%$ of the value at present with respect to the $1 \%$ obtained considering only the full pole semiperiods.

In consideration of these results, the accelerator team of Frascati accepted this as the definitive configuration to

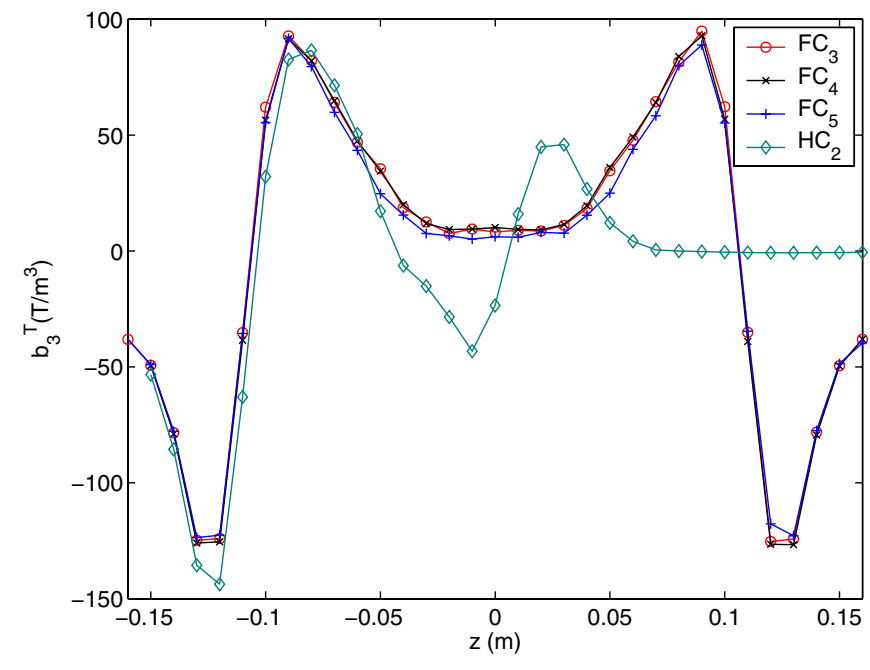

FIG. 20. (Color) Third order term of the field expansion as a function of the longitudinal axis for the semiperiods at positive $z$. In the graph for the full poles $z=0 \mathrm{~m}$ corresponds to their center, whereas for the half pole $\mathrm{HC}_{2} z=-0.16 \mathrm{~m}$ indicates the end of the semiperiod of the previous full pole $\mathrm{FC}_{5}$. 
modify the wigglers of DA $\Phi$ NE, but in the future, if necessary, also the terminal poles can be optimized to further reduce $I_{3}$ calculated in the entire wiggler. The modification of the wigglers and the measurements on the beam should be done in 2007 .

\section{CONCLUSIONS}

In periodic magnets, as undulators and wigglers, the only integrated multipoles with respect to the beam trajectory which may perturb the beam dynamics are the odd ones in the U.S. notation, because the others cancel themselves in each period of the magnets. A method to make null a specific integrated odd multipole and at the same time to reduce all the other odd orders has been presented and applied to the wigglers of the main ring of DAФNE to minimize the integrated octupole.

The simulations indicate that an opportune alternating displacement of the poles to compensate this integrated odd multipole in each semiperiod of the magnet allows to strongly reduce the integrated octupole. To minimize also the dependence of the solution on the beam-wiggler misalignment, shims have been added to the poles to improve the transverse field uniformity. In the definitive configuration the simulations show that the integrated octupole in these wigglers can be reduced to $3 \%$ with respect to the present configuration by this modification.

The modification of the wiggler according to this model should be done in 2007.

\section{ACKNOWLEDGMENTS}

I would like to thank the accelerator division of the Laboratori Nazionali di Frascati (Frascati, INFN
Laboratory) and, in particular, M. Calvetti, S. Guiducci, M. Preger, P. Raimondi, and C. Sanelli for the fruitful discussions and the possibility they gave me to work on this project. I am also grateful to W. Scandale and D. Tommasini (CERN, Geneva, Switzerland) for their support and the hints they gave me during this work and to G. Bellomo (Universitá degli Studi di Milano, Milan, Italy) for his precious comments and reviews.

[1] P. Elleaume, in Proceedings of the 1992 European Particle Accelerator Conference, Berlin.

[2] J. Safranek, C. Limborg, A. Terebilo, K. I. Blomqvist Danfysik, P. Elleaume, and Y. Nosochkov, Phys. Rev. ST Accel. Beams 5, 010701 (2002).

[3] C. Milardi, D. Alesini, G. Benedetti, S. Bertolucci, C. Biscari, M. Boscolo, S. Di Mitri, G. Di Pirro, A. Drago, A. Ghigo, S. Guiducci, G. Mazzitelli, M. A. Preger, F. Sannibale, A. Stecchi, C. Vaccarezza, M. Zobov, P. Raimondi, and E. A. Perevedentsev, in Proceedings of the 2001 Particle Accelerator Conference, Chicago.

[4] A. Battisti, S. Bertolucci, B. Bolli, S. Ceravolo, M. Incurvati, F. Iungo, M. Paris, M. Preger, P. Raimondi, C. Sanelli, F. Sardone, F. Sgamma, and M. Troiani, DAФNE technical note MM-34, 2004.

[5] Opera Vector Fields analysis (Tosca), software for electromagnetic design, version 10.509, Vector Fields Ltd., Oxford, England, Vector Fields Inc., Illinois, USA.

[6] A.S. Artamov, L. M. Barkov, V.B. Baryshev, N.S. Bashtovoy, N.A. Vinokurov, E.S. Gluskin, G. A. Korniukhin, V.A. Kochubei, G. N. Kulipanov, N. A. Mezentsev, V.F. Pindiurin, A. N. Skrinsky, and V.M. Khorev, Nucl. Instrum. Methods 177, 239 (1980). 